\title{
Original
}

\section{Estudio bibliométrico sobre tuberculosis en trabajadores de la salud}

\section{Bibliometric tuberculosis study in health care workers}

\author{
Óscar Andrés Cruz Martínez' ${ }^{1}$ Alba Idaly Muñoz Sánchez ${ }^{2-4}$ \\ 1. Universidad Nacional de Colombia. Bogotá. Colombia \\ 2. Universidad del Valle. Brasil \\ 3. Universidad de Sao Paulo. Brasil \\ 4. Facultad de Enfermería. Universidad Nacional de Colombia. Bogotá. Colombia
}

\section{Recibido: 03-08-12}

Aceptado: 05-10-12

\section{Correspondencia}

Alba ldaly Muñoz Sánchez

Facultad de Enfermería, Edificio 101, piso 9 Oficina 910

Carrera 30 n. ${ }^{\circ} 45-03$

Universidad Nacional de Colombia. Bogotá. Colombia. Sur América

Tfno: $57(1) 3165000$ ext: 17082

Fax: 57 (1)3165000 Ext: 17021

Correo electrónico: aimunozs@unal.edu.co; albaidalymunoz@gmail.com

Resumen

La tuberculosis se considera una problemática en la salud pública y representa una prioridad en los trabajadores del sector salud.

Objetivo: Realizar descripción sobre la producción científica del tema de exposición de los Trabajadores de la Salud al agente Mycobacterium Tuberculosis.

Materiales y Métodos: Se efectuó estudio Bibliométrico sistemático y organizado sobre el tema, cuantificando la producción en cuatro bases de datos científicas desde el año 2000 al 2011.

Resultados: Se realizó la revisión de 132 publicaciones principalmente generadas en países como Estados Unidos 24\%, Brasil 13\%, Inglaterra 9\%, Francia 5\% entre otros. En el caso de América Latina, los estudios en el tema son limitados. Se encontró que más del $50 \%$ de publicaciones trataban temas de incidencia y prevalencia de casos de Tuberculosis en los trabajadores de la salud, un $25,7 \%$ hablan sobre la detección de infección latente mediante prueba de Tuberculina, Interferón Gama y Genotipificación. Un 6,8\% abordaban estudios sobre métodos de control de la infección en hospitales. Los estudios correspondieron en más de un $50 \%$ a metodologías de tipo cuantitativas.

Conclusión: Las investigaciones en países en vías de desarrollo, y en particular en Colombia, son limitadas y se requiere mayor investigación hacia la formulación de políticas públicas, la vigilancia ocupacional y en la salud y seguridad en los trabajadores que cuidan la salud.

Med Segur Trab (Internet) 2012; 58 (229) 303-320

Palabras Claves DeCS; Salud Ocupacional, Tuberculosis, Trabajador de la Salud, Bibliométrica. 
Abstract

Tuberculosis is considered a public health issue and a priority in health care workers.

Objective: the scientific description of the issue of exposure of Health Workers agent Mycobacterium tuberculosis.

Materials and Methods: We performed a systematic and organized Bibliometric study on the subject, quantifying the production of four scientific databases from 2000 to 2011.

Results: We performed a review of 132 publications primarily generated in countries like the U.S. $24 \%$, Brazil 13\%, England 9\%, France 5\% among others. In the case of Latin America, studies on the subject are limited. We found that over $50 \%$ of publications addressed issues of incidence and prevalence of TB cases in health workers, $25.7 \%$ speak on the detection of latent infection by tuberculin test, Interferon Gamma and Genotyping. 6.8\% studies on methods of addressing infection control in hospitals. The studies were for more than $50 \%$ of such quantitative methodologies.

Conclusion: Research in developing countries and particularly in Colombia, are limited and more research is needed into the formulation of public policy, occupational surveillance and the health and safety in health care workers.

Med Segur Trab (Internet) 2012; 58 (229) 303-320

MeSH Keywords, Occupational Health, Tuberculosis, Healthcare Workers, bibliometrics. 


\section{INTRODUCCIÓN}

La tuberculosis (TB) ha tenido graves implicaciones en la salud pública a nivel mundial; cada año se registran 8,8 millones de casos nuevos y 1,1 millones de muertes, estimándose que un tercio de la población mundial porta de forma latente la enfermedad ${ }^{1}$. Si bien, la TB representa un interés general hacia el control de la enfermedad en la población desde la salud pública, hoy en día cobra una relevante importancia en la salud y seguridad en el trabajo en el sector salud. La TB puede considerarse un riesgo ocupacional que puede generar efectos adversos en la salud de los trabajadores que cuidan la salud. En este sentido, se ha evidenciado un interés reciente en el desarrollo de estudios investigativos, que permitan comprender y analizar dicha problemática de importancia en los trabajadores de la salud, sin embargo, la investigación misma, y los desafíos para la vigilancia ocupacional y el control de la exposición laboral aún pueden considerarse insuficientes.

En tanto la TB ha sido descrita como un riesgo ocupacional para los trabajadores de la salud, dada la posibilidad de exposición o infección de 10 a 100 veces mayor en relación a la población en general ${ }^{2-3}$. Algunos países del mundo han indicado cifras de contagios en trabajadores de la salud con tasas que varían entre los 2 a los 2.038 casos, en 100 mil trabajadores. Esta cifra es proporcionalmente distribuida, en aquellas regiones con altas incidencias de la enfermedad en el mundo; por ejemplo, los países del continente asiático cuentan con una carga de TB del 59\%, África un 26\%, el Mediterráneo 7\%, Europa un 5\% y la región de las Américas el 3\% y también, se ha evidenciado el incremento de reporte de casos de trabajadores de la salud que se contagiaron con cepas farmacorresistentes que dificulta el tratamiento de la enfermedad ${ }^{4}$.

La salud pública, y su articulación con la salud laboral juegan una relevante importancia en el análisis de la exposición laboral en los trabajadores de la salud. El sector salud puede considerarse complejo en la salud y seguridad de los trabajadores, dada la posibilidad de contacto con múltiples agentes peligrosos derivados de la exposición a sustancias químicas, contaminantes físicos, biológicos, alta carga física y mental, y condiciones organizacionales que median en el proceso salud-enfermedad en los trabajadores 5 . En el componente del ambiente físico de trabajo, se encuentra la probabilidad de exposición a los contaminantes biológicos, y en particular al agente Mycobacterium Tuberculosis, agente bacteriano que puede transmitirse a través de la vía respiratoria, favoreciendo la transmisión cruzada o "nosocomial", de pacientes infectados con TB pulmonar al personal de salud; diferentes estudios han evidenciado la exposición laboral de los trabajadores de la salud a la TB en sus escenarios de trabajo $^{6-7}$.

Por su parte, autoridades como la Organización Mundial de la Salud y la Organización Internacional del Trabajo reconocen la tuberculosis como una problemática a nivel laboral en el sector salud, y a sus trabajadores, enfermeros, médicos, auxiliares, personal de servicios generales, administrativos, estudiantes practicantes, entre otros como prioridad de intervención para cuidar su salud ${ }^{8}$.

En las instituciones de salud como hospitales y en particular en aquellos centros, donde se maneja un gran número de casos de TB, puede darse el contagio del personal al existir múltiples factores que favorecen su transmisión ambiental que median con las condiciones de salud en los individuos. Los pacientes "baciliferos" o con TB pulmonar activa sin tratamiento, al toser, estornudar, hablar, o cuando se realizan procedimientos como nebulizaciones, broncoscopias, inducción de tos, u otros, pueden generar aerosoles o "droplets" infecciosos con bacilos de 1 a 5 micras de diámetro, que pueden quedar suspendidos en los ambientes de trabajo y ser inhalados por el personal de salud". De hecho, estudios han demostrado casos de tuberculosis en trabajadores en hospitales de alta carga de enfermedad, que adquirieron cepas multidrogorresistentes (MDR-TB), y extremadamente resistentes (XDR-TB), a los medicamentos efectivos del tratamiento como la Isoniacida, la Rifampicina, entre otros ${ }^{10}$. 
En este sentido, el análisis de esta problemática no debe limitarse a la definición de causalidad, centrada en aspectos fisiopatológicos, sino que en el ámbito de la salud laboral, se requiere involucrar la salud pública, el análisis de las condiciones de trabajo los cuales permean el proceso salud-enfermedad-tuberculosis en los trabajadores; éste se ve influenciado por las características del agente causal, la bioseguridad y las condiciones de salud en los individuos y colectivos de trabajadores. Se requiere un análisis pormenorizado de del proceso de trabajo que se da en el sector salud, categorías que son necesarias observar en el estudio de la Relación Salud-Trabajo desde diferentes perspectivas y metodologías ${ }^{11}$.

En su aporte, el presente artículo abordará el estudio bibliométrico de la producción científica sobre el tema de la exposición ocupacional de los trabajadores de la salud al agente Mycobacterium Tuberculosis. Se efectuó revisión de 4 bases de datos científicas en los períodos comprendidos desde el año 2000 al 2011, con el objetivo de conocer el avance y el estado actual del conocimiento en la investigación y permita orientar el desarrollo de posteriores estudios en esta problemática de interés laboral.

El estudio bibliométrico como una estrategia de búsqueda de información sistemática y organizada, permite el análisis cuantitativo de la producción científica, en una determinada área de estudio, según país, año, método de publicación, entre otros aspectos relevantes, para conocer el contexto general y particular de la investigación en un tema ${ }^{12}$. Según Araujo, la Bibliométrica se ha considerado como una importante metodología que permite medir el nivel de conocimientos y desarrollo investigativo en un tema, que permite evidenciar la producción científica y el impacto que ha tenido la investigación en la comunidad científica, aplicable a cualquier campo del conocimiento y disciplina ${ }^{13}$.

En este sentido, se considera apropiado adoptar esta metodología en la revisión de la temática de exposición de los trabajadores de la salud al agente Mycobacterium Tuberculosis, que permita aproximarse a conocer y describir los aspectos generales más relevantes en el abordaje del objeto de estudio, en aspectos de marcado interés en la salud y seguridad en los trabajadores del sector salud y generar intervenciones.

\section{OBJETIVO}

Describir la producción científica realizada desde el año 2000 al año 2011 relacionado con el tema de la exposición de los trabajadores de la salud al agente Mycobacterium Tuberculosis.

\section{MATERIAL Y MÉTODOS}

Se realizó estudio bibliométrico sistemático y organizado cuantificando la producción de cuatro bases de datos científicas (Medline, Cochrane, Embase, Scielo), desde el año 2000 al año 2011, relacionada al tema objeto de estudio. Las bases de datos fueron seleccionadas teniendo en cuenta su amplia indexación de publicaciones de relevante valor científico en el área de la salud y seguridad en el trabajo y en tuberculosis, así como también, la publicación de artículos de carácter relevante a nivel científico en los idiomas inglés, español, portugués entre otros.

La búsqueda se efectuó consultando el lenguaje tesauro y Mesh más apropiado al tema objeto de estudio. Se utilizaron operadores Boleanos para conformar la ecuación de búsqueda. Finalmente, se realizó revisión de abstracts y títulos para verificar la pertinencia del tema objeto de estudio y la no repetitividad de publicaciones entre bases de datos.

La información se organizó en tabla de datos del programa Excel 2007, mediante los siguientes criterios de compilación: área temática, título del estudio, año de publicación, idioma de divulgación, país de procedencia, journal o revista de origen, nombre de autor(es), tipo de publicación. Para el análisis de la información se establecieron frecuencias simples y cifras porcentuales las cuales fueron calculadas a partir de la revisión y clasificación de la información recopilada. 


\section{RESULTADOS}

A continuación se presentan los resultados más relevantes en la revisión bibliométrica sobre la exposición ocupacional de los trabajadores de la salud a la Tuberculosis:

\section{Hallazgos generales de búsqueda}

Para la búsqueda bibliométrica sobre el tema de estudio, se definieron 3 descriptores en Inglés y su correspondiente en español; Occupacional Health, Tuberculosis y Healtbcare Workers aplicando el operador Boleano AND, lo cual permitiera la búsqueda simultánea de los tres términos. Fueron utilizadas limitaciones de búsqueda especificando publicaciones indexadas en el período comprendido del 01/01/2000 a cohorte del 21/04/2011. A continuación se indica el número de publicaciones relacionadas en cada base de datos:

Tabla 1. Número de Publicaciones Exposición Ocupacional a Mycobacterium Tuberculosis en trabajadores de la salud

\begin{tabular}{|c|c|c|c|}
\hline BASE DE DATOS & ECUACIÓN DE BÚSQUEDA/BOLEANOS & $\begin{array}{c}\text { NÚMERO } \\
\text { DE PUBLICACIONES }\end{array}$ & PORCENTAJE \\
\hline MEDLINE & $\begin{array}{l}\text { Occupational AND }(+) \text { Tuberculosis AND } \\
\text { [2000-2011] }\end{array}$ & 111 & $35,2 \%$ \\
\hline SCIELO & $\begin{array}{l}\text { Occupational AND }(+) \text { Tuberculosis AND } \\
\text { [2000-2011] }\end{array}$ & 24 & $7,61 \%$ \\
\hline EMBASE & $\begin{array}{l}\text { Occupational AND (+) Tuberculosis AND } \\
\text { Healthcare AND workers AND [2000-2011] }\end{array}$ & 174 & $55,2 \%$ \\
\hline COCHRANE & $\begin{array}{l}\text { Occupational AND }(+) \text { Tuberculosis AND } \\
\text { [2000-2011] }\end{array}$ & 6 & $1,9 \%$ \\
\hline TOTAL & & 315 & $100 \%$ \\
\hline
\end{tabular}

Estudio Bibliométrico: Exposición Ocupacional a la Tuberculosis en Trabajadores de la Salud (2000-2011).

\section{- Consideraciones:}

Teniendo en cuenta la tabla anterior, se evidenció un mayor número de publicaciones relacionadas al objeto de búsqueda en la base de datos Embase con un número igual a 174 o (Núm.=174), correspondiente al (55,2\%); seguida por Medline con $n=111(35,2 \%)$; Scielo con Núm.=24 (7,61\%) y Cochrane con Núm.= $6(1,9 \%)$, para un total de 315 publicaciones.

Se evidenció que Embase cuenta con un gran número de Abstracts y artículos Full Text sobre el tema, los cuales también se encuentran disponibles en otras bases de datos como Medline, Cochrane y Scielo. Luego de la revisión general de abstracts, títulos, y autores en las 315 publicaciones de la búsqueda inicial, se realizó un filtro para verificar la relación de pertinencia con el tema objeto de estudio. También se verificó la no repetitividad de publicaciones en las 4 bases de datos seleccionadas, y de allí fueron seleccionadas 132 publicaciones desde las cuales se presentan los siguientes resultados:

\section{Clasificación temática del tema de estudio}

Realizando la revisión de las 132 publicaciones fueron indagados los temas generales y se realizó la clasificación teniendo en cuenta la frecuencia de resultados. Se evidenció de forma general que en los artículos seleccionados se abordan diferentes temas relacionados a la exposición a la tuberculosis en trabajadores de la salud. 
Tabla 2. Clasificación Temática del tema Exposición Ocupacional a la Tuberculosis en Trabajadores de la Salud

\begin{tabular}{|c|c|c|}
\hline ÁREA TEMÁTICA & $\mathbf{N}=$ Frecuencia & Porcentaje \\
\hline $\begin{array}{l}\text { 1. Epidemiología de casos de TB latente y activa en trabajadores de la } \\
\text { Salud y los Factores asociados al contagio. }\end{array}$ & 40 & $30,3 \%$ \\
\hline $\begin{array}{l}\text { 2. Pruebas de Detección de la infección latente: TST, TSPOT-TB, INF- } \\
\text { Gamma (IGRA). }\end{array}$ & 34 & $25,7 \%$ \\
\hline $\begin{array}{l}\text { 3. Métodos de control de la exposición al agente M. Tuberculosis en } \\
\text { hospitales: (Ventilación, Filtros HEPA, Luz UV, Aislamiento, Uso de } \\
\text { Respiradores } \mathrm{N}^{\circ} \text { 95). }\end{array}$ & 9 & $6,8 \%$ \\
\hline $\begin{array}{l}\text { 4. Perfil de Riesgo de exposición a la TB según Profesión en los } \\
\text { trabajadores de la salud. }\end{array}$ & 9 & $6,8 \%$ \\
\hline $\begin{array}{l}\text { 5. Pruebas Moleculares de Genotipificación de M. Tuberculosis en } \\
\text { trabajadores de la salud. }\end{array}$ & 5 & $3,8 \%$ \\
\hline $\begin{array}{l}\text { 6. Exposición a casos de Multidrogoresistencia MDR-TB y casos de } \\
\text { Coinfección TB-VIH/SIDA. }\end{array}$ & 4 & $3,0 \%$ \\
\hline 7. Tratamiento de la infección latente y activa en trabajadores de la salud. & 3 & $2,3 \%$ \\
\hline $\begin{array}{l}\text { 8. Factores Inmunológicos involucrados en la resistencia al agente } \\
\text { M. Tuberculosis. }\end{array}$ & 2 & $1,5 \%$ \\
\hline 9. Otras temáticas. & 26 & $19,7 \%$ \\
\hline TOTAL & 132 & $100 \%$ \\
\hline
\end{tabular}

Fuente: Estudio Bibliométrico: Exposición Ocupacional a la Tuberculosis en Trabajadores de la Salud (2000-2011)

\section{Clasificación general por temas}

- Epidemiología de casos de TB en trabajadores de la salud.

Se evidenció en la clasificación según área temática de los 132 artículos seleccionados, el abordaje de diferentes temas relacionados a la exposición a la tuberculosis en trabajadores de la salud. En el análisis de la información, se identificó con mayor frecuencia, estudios epidemiológicos de prevalencia e incidencia de casos de la enfermedad en trabajadores de la salud con un número de 40 publicaciones (Núm.=40), correspondientes al (30,3\%) del $100 \%$ de las publicaciones revisadas. En esta categoría se evidenciaron investigaciones que relacionaban la probabilidad de contagio y de la infección tuberculosa asociada a condiciones de inmunosupresión, terapia con corticoesteroides, comorbilidades como Diabetes, Cáncer, uso de alcohol, tabaco, y factores laborales relacionados al estrés, el trabajo nocturno, exposición a químicos, cito estáticos, entre otros.

\section{- Detección de Infección latente por tuberculosis y pruebas moleculares de identificación.}

La segunda temática mencionada frecuentemente en la revisión bibliométrica fue la publicación de estudios que trataban la sensibilidad y especificidad de pruebas de detección de la infección latente por tuberculosis en trabajadores de la salud, con un número de Núm.=34 publicaciones (25,7\%). Los artículos abordan frecuentemente estudios de identificación de infección latente mediante la aplicación de la TST o PPD (Tuberculina) en trabajadores de la salud, y menor número de estudios acerca de pruebas de estimulación por antígenos específicos de $M$. tuberculosis a través de Quantiferón Gama o QTF-G y la técnica T-SPOT.TB conocida como ensayo de liberación de Interferón gama en sangre periférica o INF- Gama (IGRA). Estas han sido frecuentemente realizadas en países como Estados Unidos (EEUU), Inglaterra, Francia y Brasil para identificación con alta sensibilidad y especificidad de casos de TB latente en trabajadores de la salud.

Se evidenciaron publicaciones relacionadas a pruebas moleculares de identificación de infección por Mycobacterium Tuberculosis, con un número de Núm.=5 (3,8\%). Dichos 
estudios trataban temáticas de Genotipificación de casos de Mycobacterium Tuberculosis en trabajadores de la salud, a través del análisis del Polimorfismo genético de la Mycobaceria con los métodos de secuencias RFLP- subunidad IS6110, y la técnica conocida como Spolygotyping, que rastrean la transmisión cruzada de cepas específicas de tuberculosis mediante análisis filogenético. Estos han sido realizados en países desarrollados como EEUU, Inglaterra, Francia y Dinamarca, y puede demostrar la transmisión entre mismas cepas del caso índice.

\section{- Métodos de control de la exposición al agente Mycobacterium Tuberculosis.}

Se destacan también temáticas sobre métodos de control de la exposición al agente Mycobacterium Tuberculosis con Núm.=9 publicaciones correspondiente al (6.8\%), efectuados en hospitales de Alemania, Canadá, Suiza, EEUU, Brasil y Perú, que tratan abordajes cuantitativos de alcance descriptivo y analíticos. Se evidenció un estudio en Perú, que desarrollo un ensayo experimental en un hospital en el cual se aplicó un modelo de control de la infección tuberculosa intrahospitalaria versus métodos de control ambiental.

En la revisión bibliométrica también fueron encontrados estudios cuantitativos sobre los métodos de control, en temas como sistemas de ventilación natural y artificial, la correlación entre el número de recambios aire/hora y el contagio, así como también, estudios relacionados al riesgo de infección tuberculosa en salas de aislamientos y de procedimientos respiratorios, la evaluación del uso de elementos de protección personal como la mascarilla N-95 y controles administrativos relacionados al aislamiento de casos.

En relación al tema de métodos de control, se evidenció una publicación desarrollada sobre el tema de evaluación del riesgo ocupacional basado en la positividad de la TST, el viraje positivo de la Tuberculina, relacionado a la epidemiologia de casos de TB, la oportunidad diagnóstica, de tratamiento y las tasas de curación de pacientes del programa entre otras variables que influyen en el contagio. Se resalta de manera importante el análisis epidemiológico de los casos, con las tasas de incidencia de TB en la comunidad.

\section{- Perfil de riesgo de exposición en los trabajadores de la salud.}

Se identificaron al respecto estudios que indican el perfil de riesgo de contagio según categoría profesional en los trabajadores de la salud con un número de Núm.=9 $(6,8) \%$ publicaciones. De estos existen en mayor frecuencia Núm.=3 que trataban la incidencia de tuberculosis en personal de enfermería, Núm. 2 en profesionales de medicina, $\mathrm{n}=1$ de personal de laboratorio, Núm. =1 en radiología, Núm. $=1$ en trabajadores de Urgencias, Núm.=1 en otros trabajadores de la salud, quienes pueden representar un alto riesgo de contagio y de desarrollar TB activa. Se encontraron $n=2$ publicaciones correspondientes al $(1,5 \%)$, que identificaron las salas de autopsia, $\mathrm{y} n=2$ publicaciones $(1,5 \%)$ sobre estudios realizados en salas de urgencia y unidades de pediatría identificándose como zonas de alto riesgo de transmisión de TB.

Así mismo, Núm.= 3 estudios $(2,3 \%)$ indagaron sobre factores inmunológicos asociados al contagio, relacionados a casos de proliferación de la producción de Interferón IFN-gamma, interleukin IL-5 y 4 entre otros, realizados en Francia y EEUU como marcadores de contacto y de infección. En relación a las condiciones de inmunosupresión un estudio trató el tema del aumento de la probabilidad de desarrollo de la infección latente a enfermedad activa en el personal de la salud, en casos de confección TB-VIH/ SIDA y en trabajadores de la salud, estudios referenciados de España, Italia y EEUU.

En relación a esta temática se evidenciaron $n=4$ estudios $(3 \%)$, que relacionaban la exposición de los trabajadores de la salud a casos de Tuberculosis Farmacorresistentes y de Coinfección de Tuberculosis VIH/SIDA, en los cuales se utilizó el seguimiento al viraje de la prueba TST, mediante estudios prospectivos y retrospectivos. Se identificó un estudio relacionado a casos de alta prevalencia de transmisión de cepas de Tuberculosis MDR-TB, y XDR-TB en hospitales caracterizados por la alta carga de atención de pacientes de TB y VIH/SIDA en Sudáfrica. 
- Otras temáticas relacionadas a la exposición laboral a la M. Tuberculosis en el sector salud.

En relación a otras temáticas de investigación en el tema de exposición laboral al agente Mycobacterium Tuberculosis se evidenciaron estudios relacionados con accidentes por inoculación percutánea de cepas de $M$. Tuberculosis, bioseguridad en la exposición a cepas MDR-TB, conocimientos actitudes en el personal, un estudio sobre el tratamiento profiláctico de infección latente con Isoniacida en el personal de salud, y uno sobre reacciones adversas a la aplicación de la prueba TST.

\section{Clasificación según año de las publicaciones}

En relación a la clasificación según año de publicación de la producción científica en el tema de estudio, se tuvo en cuenta como criterio de inclusión, las publicaciones indexadas desde el año 2000 al año 2011, que permitieran cuantificar la producción según años. Se obtuvieron los siguientes resultados (ver Gráfico n. ${ }^{\circ}$ ).

Gráfico 1. Año de publicación

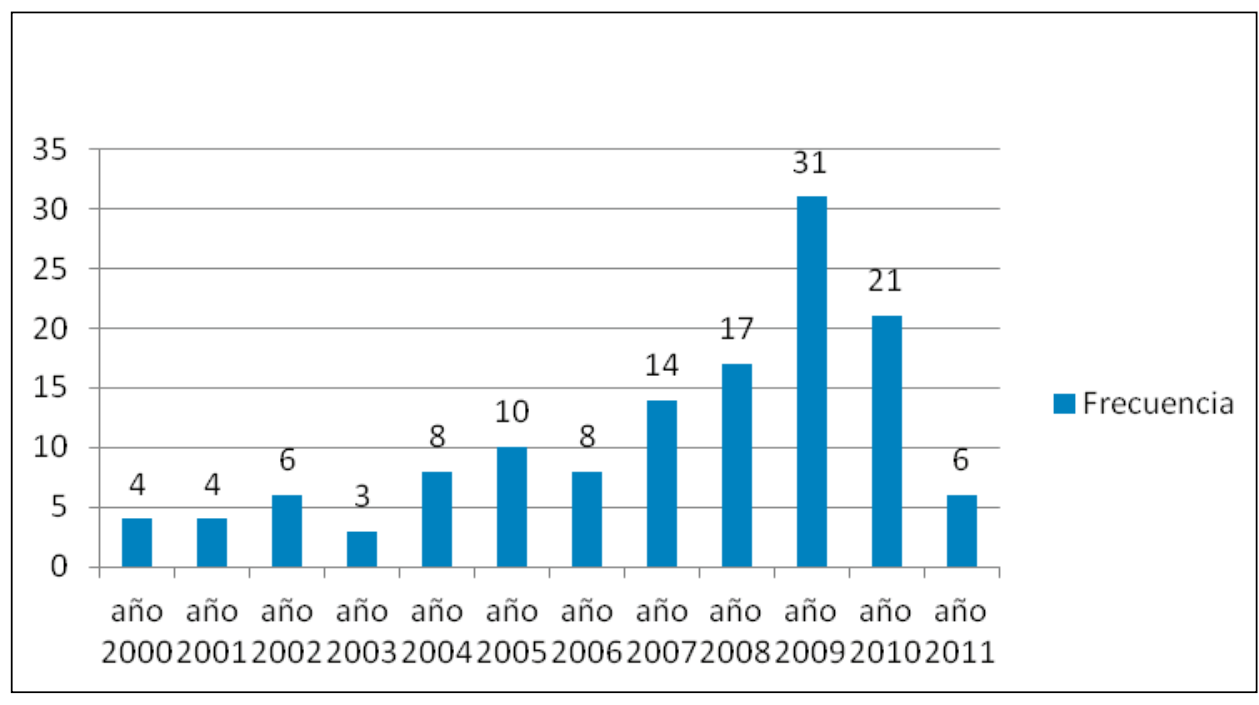

Fuente: Revisión Bibliométrica: Exposición Ocupacional a la Tuberculosis en Trabajadores de la Salud (2000-2011)

En relación al número de publicaciones divulgadas en el período (2000 al 2011), se evidenció un número máximo en el año 2009 con Núm.=31 publicaciones correspondiente al $23,4 \%$, seguido por el año 2010 con Núm.=21 (15,9\%), y al cohorte de abril de 2011 se contaban con 6 publicaciones correspondiente al $(4,5 \%)$.

\section{Clasificación de publicaciones según tipo}

Se evidenció de forma general que el tipo de publicaciones más frecuentes se desarrollan mediante un abordaje cuantitativo con alcance descriptivo Núm.=66 publicaciones equivalente a (50\%), estos trataban de forma general la incidencia y prevalencia de casos de la infección por tuberculosis en los trabajadores de la salud, seguido por artículos de revisión de tema Núm.= 26 correspondiente al (20\%) que involucran la revisión de factores asociados al contagio por tuberculosis en el personal de la salud.

Sobre artículos cuantitativos-analíticos se evidenciaron Núm.=20 publicaciones con el (15\%), que tratan ensayos comparativos entre pruebas de infección latente por tuberculosis, TST, y T-SPOT.TB, así como también, estudios de casos y controles relacionados a la efectividad de métodos de control de la infección tuberculosa en hospitales de EEUU.

Se encontraron dos investigaciones con un enfoque cualitativo de investigación correspondiente al (1\%), las cuales describían los factores de influencia en el cuidado de la salud en los trabajadores expuestos a TB y la percepción del tratamiento en la exposición ocupacionales en la infección en EEUU y Francia. 


\section{Clasificación según país de origen e idioma}

Se encontró en relación al número de publicaciones por país sobre el tema objeto de estudio, que los Estados Unidos (EEUU) cuentan con un amplio desarrollo de estudios Núm.= (30) correspondientes al (24\%), seguido por Brasil con $n=16(13 \%)$, Inglaterra con $n=14(11 \%)$ y Francia con $n=11$ (9\%). Dentro de los países latinoamericanos diferentes a Brasil se destacan México con Núm.= 3 publicaciones que corresponde al (2\%), y Perú con $n=6$ publicaciones desarrolladas en conjunto con otros países, estos últimos relacionados a temáticas sobre incidencia y prevalencia de casos, y el control de la infección tuberculosa (ver Tabla n. ${ }^{\circ}$ 3)

Tabla 3. Frecuencia de publicaciones por país

\begin{tabular}{|c|c|c|}
\hline PAÍS & FRECUENCIA & PORCENTAJE \\
\hline EEUU & 30 & $24 \%$ \\
\hline BRASIL & 16 & $13 \%$ \\
\hline INGLATERRA & 14 & $11 \%$ \\
\hline FRANCIA & 11 & $9 \%$ \\
\hline ITALIA & 7 & $5 \%$ \\
\hline ESPAÑA & 5 & $4 \%$ \\
\hline ALEMANIA & 4 & $3 \%$ \\
\hline JAPÓN & 3 & $2 \%$ \\
\hline MÉXICO & 3 & $2 \%$ \\
\hline POLONIA & 3 & $2 \%$ \\
\hline CHINA & 3 & $2 \%$ \\
\hline PERÚ & 3 & $2 \%$ \\
\hline PORTUGAL & 3 & $2 \%$ \\
\hline SUIZA & 3 & $2 \%$ \\
\hline TURQUÍA & 1 & $1 \%$ \\
\hline ARGENTINA & 1 & $1 \%$ \\
\hline BÉLGICA & 1 & $1 \%$ \\
\hline CANADÁ & 1 & $1 \%$ \\
\hline CHILE & 1 & $1 \%$ \\
\hline DINAMARCA & 1 & $1 \%$ \\
\hline EEUU-PERÚ & 1 & $1 \%$ \\
\hline EEUU-INDIA & 1 & $1 \%$ \\
\hline INGLATERRA-ALEMANIA & 1 & $1 \%$ \\
\hline INGLATERRA-PERÚ & 1 & $1 \%$ \\
\hline FRANCIA-BRASIL & 1 & $1 \%$ \\
\hline FRANCIA-COREA & 1 & $1 \%$ \\
\hline INDIA & 1 & $1 \%$ \\
\hline IRÁN & 1 & $1 \%$ \\
\hline IRLANDA & 1 & $1 \%$ \\
\hline ITALIA-PERÚ & 1 & $1 \%$ \\
\hline NIGERIA & 1 & $1 \%$ \\
\hline RUSIA & 1 & $1 \%$ \\
\hline FINLANDIA & 1 & $1 \%$ \\
\hline THAILANDIA & 1 & $1 \%$ \\
\hline COLOMBIA & 1 & $1 \%$ \\
\hline TOTAL & 132 & $100 \%$ \\
\hline
\end{tabular}

Fuente: Revisión Bibliométrica: Exposición Ocupacional a la Tuberculosis en Trabajadores de la Salud (2000-2011) 
Para el caso de Colombia se evidenció un artículo relacionado con incidencia de positividad de infección latente en estudiantes, el cual correspondió a un estudio transversal para evaluar la respuesta de la TST, en estudiantes del área de la salud (medicina, odontología, enfermería y bacteriología), en una universidad obteniendo como resultados la reacción positiva a su aplicación.

\section{Publicación según idioma}

En relación al idioma, la mayoría de publicaciones se encontraban en inglés con Núm.= 90 artículos correspondientes a el 68\%, seguido por el Español con Núm.= 16 publicaciones (12\%), y el Portugués con Núm.= 12 artículos que equivalente al 9\%. Otros idiomas de referencia de las publicaciones referenciadas fue francés con un $5 \%$, italiano $2 \%$, japonés $2 \%$, polaco $2 \%$, alemán $1 \%$, que concuerda al país de origen de las publicaciones (ver Tabla n. ${ }^{\circ}$ ).

Tabla 4. Idioma de Publicación Exposición Ocupacional a la Tuberculosis en Trabajadores de la Salud

\begin{tabular}{lc}
\hline \multicolumn{1}{r}{ IDIOMA } & N=NÚMERO \\
\hline Inglés & 90 \\
Español & 16 \\
Portugués & 12 \\
Francés & 6 \\
Italiano & 3 \\
Polaco & 2 \\
Japonés & 2 \\
Alemán & 1 \\
\multicolumn{1}{c}{ Total } & 132 \\
\hline
\end{tabular}

Fuente: Estudio Bibliométrico: Exposición Ocupacional a la Tuberculosis en Trabajadores de la Salud (2000-2011)

\section{Artículos según revista de publicación}

En relación a las revistas de publicación de los artículos en las bases de datos consultadas, se evidenció que de mayor a menor número de publicaciones fueron; la International Journal of Tuberculosis and Lung Disease con 9 publicaciones (6,8\%), la BMC Infectious Diseases con 7 publicaciones (5,3\%), la Journal Of Occupational Medicine And Toxicology con 6 publicaciones (4,5\%), Infection Control and Hospital Epidemiology con 5 publicaciones $(3,7 \%)$ y otras revistas con un artículo cada una conformando un $66,4 \%$ (ver Tabla n. ${ }^{\circ}$ ). 
Tabla 5. Revistas con publicaciones sobre Tuberculosis en trabajadores de la salud

\begin{tabular}{lcc}
\hline \multicolumn{1}{c}{ REVISTA } & FRECUENCIA & PORCENTAJE \\
\hline International Journal of Tuberculosis and Lung & 9 & $6,8 \%$ \\
$\quad$ Disease & 7 & \\
BMC Infectious Diseases & 6 & $5,3 \%$ \\
Journal of Occupational Medicine And Toxicology & 5 & $4,5 \%$ \\
Infection Control and Hospital Epidemiology & 4 & $3.7 \%$ \\
Rev. salud pública & 3 & $3,0 \%$ \\
Biomédica & 3 & $2,3 \%$ \\
Clinical Infectious Diseases & 3 & $2,3 \%$ \\
Emerging Infectious Diseases & 3 & $2,3 \%$ \\
Journal of Hospital Infection & 3 & $2,3 \%$ \\
Plos One & 3 & $2,3 \%$ \\
Scandinavian Journal of Infectious Diseases & 83 & $2,3 \%$ \\
Otras revistas & $\mathbf{1 3 2}$ & $66,4 \%$ \\
$\quad$ TOTAL & & $\mathbf{1 0 0} \%$ \\
\hline
\end{tabular}

Fuente: Estudio Bibliométrico: Exposición Ocupacional a la Tuberculosis en Trabajadores de la Salud (2000-2011)

\section{DISCUSIÓN}

La investigación en tuberculosis a través de los últimos años ha abordado diferentes estudios desde la perspectiva de la salud pública. Sin embargo, relacionado al tema de exposición laboral en el sector salud, ha evidenciado el desarrollo reciente de diversas temáticas, las cuales en su mayoría corresponden a estudios de abordajes cuantitativos de alcance descriptivos, relacionando la epidemiológica de casos de la enfermedad activa o latente y pruebas de detección de la infección en su mayoría desde países desarrollados ${ }^{14}$.

$\mathrm{Al}$ respecto, Özsoy y otros en investigaciones en hospitales indicaron que el riesgo de exposición ocupacional de los trabajadores de la salud a la tuberculosis es alto en el personal que trabaja en lugares donde existen salas de patología y cirugía, correlacionándolo el tiempo de permanencia y en la posibilidad de generación de aerosoles infecciosos durante la disección de tejidos ${ }^{15}$. Otros autores han mencionado posibilidad de contagio en salas de urgencias, pediatría, salas de espera, laboratorio clínico los cuales han permitido definir la prevalencia e incidencia de casos de infección en los trabajadores de la salud. Incluso, se han utilizado para clasificar zonas de mayor riesgo de transmisión de la enfermedad, basándose en las pruebas para la identificación de la infección latente (Tuberculina), caracterizando el riesgo de exposición que se da en la atención en salud en las instituciones sanitarias ${ }^{16-17}$.

Por su parte, un estudio latinoamericano fue desarrollado en hospitales de Brasil para identificar el nivel de exposición de los trabajadores del sector salud al agente Mycobacterium Tuberculosis. Al respecto, se observó que el 63,1\% de trabajadores de cuatro hospitales presentó positividad con una tasa de conversión de $10,7 \%$ en la PPD $^{18}$. Otros estudios en relación a la categorización de profesiones de riesgo han identificado que los hospitales sin medidas de control de la infección por Tuberculosis, muestran una detección de positividad de PPD aumentada en profesionales de enfermería de hasta el $69,5 \%$, situación compleja en la salud y seguridad laboral. Estos profesionales dado al proceso de atención directo que realizan a los pacientes, el desarrollo de procedimientos y condiciones particulares de trabajo pueden exponerse a un alto riesgo de infección y desarrollo de formas activas de la enfermedad ${ }^{19}$. 
Bonifacio y otros, en relación a otras categorías profesionales mencionaron que el personal médico y los estudiantes de medicina, tienen mayor riesgo de infección o de desarrollar la enfermedad activa, evidenciando una tasa anual de infección latente para internos y médicos en contacto con pacientes diagnosticados con la enfermedad de 17\% y un $2 \%$ respectivamente ${ }^{20}$. De igual manera, se han documentado casos en otras categorías de profesiones en la salud como paramédicos, personal de terapia respiratoria y en auxiliares de enfermería encargados de desarrollar procedimientos que involucran la generación de aerosoles ${ }^{21}$. Los estudios en otros trabajadores de la salud como personal de servicios generales, limpieza, mantenimiento, personal administrativo, ingenieros sanitarios, entre otros que trabajan en hospitales, son limitados.

En México estudios referencian una positividad basal de la prueba de Tuberculina o PPD cerca del $40 \%$ en trabajadores de la salud, con una cifra de conversión a PPD positivo en $43.3 \%$, mencionando que los trabajadores previamente no infectados llegan a contagiarse dentro del hospital en los primeros dos años, derivado en fallas en la aplicación de normas de bioseguridad, el diagnóstico y tratamiento tardío de los pacientes, entre otros aspectos ${ }^{22}$.

La investigación en el tema de la exposición laboral a la tuberculosis en trabajadores de la salud en un sentido amplio, se ha enfocado al estudio de la prevalencia e incidencia de casos de TB latente mediante la utilización de la PPD o Tuberculina que identifica infección previa. Sin embargo, algunos autores han demostrado su baja especificidad en relación, a la posibilidad de generar falsos positivos o negativos por reacción cruzada con antígenos previos a la vacunación con BCG (Bacillus de Calmette y Guérin), casos paucibacilares de la TB, estados de inmunosupresión o mala técnica de aplicación ${ }^{23}$.

Para la identificación de la infección latente por tuberculosis existen otros estudios y técnicas de abordaje analítico que han demostrado mayor sensibilidad y especificidad como el Quantiferón Gama o QTF-G y la técnica T-SPOT.TB ensayo de liberación de Interferón gama en sangre periférica o INF- Gama (IGRA), las cuales pueden ser de gran utilidad en la identificación oportuna de casos de infección latente en trabajadores de la salud y en la vigilancia ocupacional de los trabajadores en hospitales ${ }^{24-25}$.

En el caso de Colombia, Arbeláez y otros han indagado la positividad de la reacción de la prueba de Tuberculina en estudiantes de las áreas de la salud, obteniéndose una asociación $(\mathrm{P}=0,007)$, entre la reacción positiva de la PPD y la BCG ampliamente utilizada en el país en los esquemas de vacunación para niños menores a un año ${ }^{26}$. En este sentido para el caso particular del país la investigación es limitada y se requiere la utilización de otros ensayos para la identificación de TB latente mediante pruebas como el Quantiferón Gama y otros en la vigilancia y control de la exposición.

En el estudio de la exposición ocupacional también se incluyen estudios que han tratado pruebas moleculares de identificación de la infección tuberculosa en trabajadores de la salud. Países como Estados Unidos, Inglaterra y otros del continente europeo, entre otros, han utilizado pruebas de Genotipificación de Mycobacterias mediante metodología de análisis genómico denominado RFLP IS6110, y la técnica conocida como Spolygotypin que realizan identificación del ácido desoxirribonucleico bacteriano (ADN) mediante metodología de cadena de reacción de polimerasa (PCR), las cuales son técnicas que han demostrado la posibilidad de obtener una identificación genotípica de las cepas de Mycobacterium $^{27}$.

Desde el contexto de la salud laboral la Genotipificación de Mycobacterias se convierten en herramientas importante para incluso distinguir el origen de la enfermedad laboral o común, dado a que se puede realizar un análisis de especies a nivel filogenético de Mycobacterias, en los casos de trabajadores de la salud contagiados con pacientes, permitiendo así demostrar la causalidad ocupacional ${ }^{28-29}$.

En relación a la identificación de la exposición al agente Mycobacterium Tuberculosis, si bien se ha vislumbrado el desarrollo de diferentes técnicas moleculares, en países desarrollados, en los países en "vías de desarrollo", como Colombia, se requiere el 
fortalecimiento de los sistemas de vigilancia epidemiológicos ocupacionales, la red de laboratorios de referencia en el desarrollo de dichas pruebas, y el fomento de la investigación aplicada, que posibilite el reconocimiento de casos de dicha enfermedad en el estudio del origen ocupacional en el sector salud. Según afirma Sánchez Torrago, la investigación en los países de forma general ha estado mediada por el desarrollo económico y social, en la cual se destacan las brechas entre países en vías de desarrollo y países desarrollados evidenciando dificultades relacionadas al acceso a tecnologías, políticas de inversión económica, fuentes de financiación para la investigación ${ }^{30}$.

Otra temática de gran importancia en la revisión bibliométrica y la cual ha sido poco estudiada la constituyen la identificación de casos de trabajadores de la salud que adquirieron formas de Tuberculosis Resistente a Fármacos la cual ha sido documentada, en hospitales de alta carga y admisión de pacientes con la enfermedad ${ }^{31}$. En el mundo existen aproximadamente 0.65 millones de casos de Tuberculosis Multidrogorresistentes o (MDR-TB), a los medicamentos de primera línea efectivos contra la enfermedad (Isoniacida y Rifampicina). También, se estiman más de 25.000 casos de Tuberculosis Extremadamente resistente o (XDR-TB), tanto a fármacos de primera y segunda línea (Fluoroquinolonas y amino-glucósidos, etc.), y en el año 2007 se reportaron casos de Tuberculosis Totalmente Resistente (TDR-TB), una cepa incurable y altamente letal de la enfermedad que fue detectada desde el 2007, y lo cual complejiza acciones de diagnóstico, tratamiento y control de la infección tuberculosa a nivel laboral en las instituciones de salud ${ }^{32}$.

En la revisión bibliométrica se identificaron estudios relacionados a los factores asociados a la reactivación de la enfermedad desde las condiciones de salud en los trabajadores de la salud; Rafiza y otros identificaron la edad mayor a 35 años [9.49 (IC: 2,$22 ; 40,50$ )], así como, la historia de la vida en la misma casa con familiares cercanos o amigos que tenía tuberculosis activa [8.69 (IC: 3,00; 25,18)], el hecho de trabajar en enfermería [4.65 (IC: 1,$10 ; 19,65)$ ] y pertenecer al sexo masculino como predictores de contagio $^{33}$.

Por otro lado, Sepkowitzy y otros ${ }^{34}$ indicaron que los trabajadores de la salud quienes han presentado algún estado inmunosupresor ocasionado por el VIH/SIDA un fuerte reactivador de la tuberculosis; algunos aspectos asociados a factores extralaborales y de carga mental del trabajo en el sector salud, podrían estar asociados a la disminución de la capacidad inmunológica agente-huésped. Autores como Murray destacan, que los estados inmunosupresores pueden reactivar la enfermedad, comúnmente asociada a casos de existencia de comorbilidades inmunosupresoras como por ejemplo la transcurrida en la Diabetes Mellitus, Cáncer, pacientes consumidores de alcohol, cigarrillo, en desnutrición, bajo tratamiento de corticoesteroides, estrés, entre otros, como factores relacionados que aumentan la vulnerabilidad de exposición y desarrollo de la tuberculosis que requieren análisis desde las condiciones individuales de salud en el estudio de la exposición ${ }^{35}$.

Otra de las temáticas relevantes en el análisis del tema de exposición laboral a la tuberculosis lo constituye la identificación de factores relacionados a las condiciones de trabajo. Las condiciones de trabajo son definidas como el "Conjunto de aspectos singulares propios de cada trabajador como sujeto histórico-social, así como las condiciones intralaborales (ambiente, tarea, organización) y extralaborales en el desarrollo del proceso de trabajo, inmerso en un entorno y contexto definido, que pueden generar o no procesos de salud-enfermedad o de bienestar" ${ }^{36}$.

En tanto, las condiciones de trabajo en la posibilidad de exposición al agente Mycobacterium Tuberculosis puede involucrar factores de exposición a la infección o desarrollo de la enfermedad en los trabajadores de la salud; en la revisión bibliométrica se evidencian estudios que han identificado predisposición al contagio en los trabajadores. Según Nery y Bertolozzi, quienes desarrollaron un estudio en personal de enfermería en un Hospital de Brasil, identificaron factores relacionados al trabajo y la organización del trabajo. Se puede aumentar la vulnerabilidad de contagio en los trabajadores de los turnos nocturnos, los dos primeros años de trabajo, las jornadas laborales mayores a 12 
horas diarias, el trabajar como auxiliar de enfermería fueron fuertes predictores que podrían estar implicados en la reactivación de la infección latente ${ }^{37}$. Desde las condiciones ambientales de trabajo las malas condiciones de ventilación, el hacinamiento de los puestos de trabajo y la ausencia de recambios de aire pueden aumentar el riesgo de transmisibilidad de la tuberculosis. Según modelos generados por Riley-Wells en los tiempos que permanecen suspendidas partículas infecciosas de tuberculosis en el ambiente $^{38}$. Otro estudio indagó el riesgo relativo de infección por TB en hospitales, evidenciado que fue 3,4 veces mayor en trabajadores que se encontraban en salas que tenían menos de dos recambios de aire hora, y en aquellos que no contaban con sistemas de ventilación natural, o ductos para la filtración de extracción de aire ${ }^{39}$.

Desde el componente del control de la infección tuberculosa en hospitales, en la revisión bibliométrica sobre la exposición a la tuberculosis en trabajadores de la salud se identificó en hospitales latinoamericanos factores relacionados a la exposición en el ambiente de trabajo derivado del déficit de medidas de bioseguridad. Estudios en hospitales han realizado modelos de evaluación con diferentes métodos de control ambiental de la infección tuberculosa como sistemas de ventilación natural y artificial, la utilización de luz ultravioleta en la parte alta de salas de aislamiento, los filtros de alta eficiencia o HEPPA, medidas administrativas de aislamiento de casos, y de protección individual como el uso de mascarillas $\mathrm{N}-95^{40}$.

Un estudio realizado por Echanove y otros, en un Hospital de Lima (Perú), en el cual se aplicó un modelo de ventilación de aire y de ionización de aire mediante luz ultravioleta, en los cuartos de pacientes diagnosticados con TB y VIH, adaptó en la azotea del hospital un cuarto con "Guinea Pigs" susceptibles a la infección por TB. En este estudio se aplicaron diferentes métodos de control de eliminación del agente bacteriano y seguimiento mediante pruebas histopatológicas y del Interferón Gamma, identificándose en un 35\% lesiones infecciosas en contactos. Se logro reducción del riesgo en un 14\% (43/303) mediante ionización, 9.5\% (29/307) por luz UV ( $\mathrm{p}<0.0001$ comparado con el grupo control). Los modelos analíticos indicaron una reducción de infección por TB de hasta el 51\% usando técnica luz UV "Upper-Room Ultraviolet Light”, y mediante ionización del aire en un $60 \%{ }^{41}$.

Otros estudios en el control de la infección tuberculosa han indagado el fomentar los conocimientos sobre la enfermedad ${ }^{42}$, la percepción de riesgo al contagio y la bioseguridad $^{43}$, medidas administrativas en el control de la infección, el aislamiento de casos, el uso de la mascarilla N95, todas estas medidas, recomendadas y promulgadas por organismos como el Centro de Control de Enfermedades de Atlanta (CDC), para los centros de atención sanitaria ${ }^{44}$.

En Colombia y otros países se identifica el déficit de medidas de infraestructura física en el control de la infección tuberculosa en centros de atención a pacientes con tuberculosis. En este sentido, esta problemática requiere ser ampliamente desarrollada mediante estudios descriptivos, analíticos, la generación de instrumentos de identificación validados que permitan conocer factores de exposición a la tuberculosis en trabajadores de la salud de hospitales.

Las investigaciones en la identificación y el control de la exposición al agente Mycobacterium Tuberculosis en el sector salud se considera complejo, en el cual se requiere el abordaje interdisciplinario desde la higiene industrial, la epidemiológica, la medicina y enfermería laboral entre otros. Tal como lo plantea Lozada y Muñoz ${ }^{45}$, las investigaciones sobre la relación salud-trabajo en los trabajadores, ameritan el análisis integral de las condiciones de trabajo y salud, en aquellos aspectos que componen la dinámica histórico-social del trabajador, la esfera intralaboral y extralaboral relacionada a la ejecución del proceso de trabajo, inmerso en un entorno en un tiempo determinado. Así mismo se reconocen las necesidades de la generación de instrumentos y herramientas psicométricamente válidas y confiables en el abordaje de dicha problemática, desde metodologías subjetivas y objetivas, que permitan identificar perfiles de vulnerabilidad y de riesgo de exposición a la TB en los trabajadores de la salud. 


\section{CONCLUSIONES}

La tuberculosis al ser considerada como una enfermedad de prioridad en salud pública, ha sido descrita en diferentes investigaciones como un agente de relevancia en la salud laboral. Sin embargo, se hace necesario profundizar en diferentes abordajes metodológicos analíticos y de abordaje cualitativo que permitan obtener una mayor comprensión en la problemática de la exposición laboral a la tuberculosis en el sector salud y ampliar las tendencias de investigación en tema de estudio.

De igual manera, se reconoce la necesidad de fortalecer y fomentar estudios en países en vías de desarrollo donde confluyen problemáticas importantes relacionadas a la alta carga de incidencia y prevalencia de casos de la enfermedad, en su determinación social ligada a condiciones precarias de vida en la población. La investigación en tuberculosis a nivel laboral debe partir del estudio integral de la relación salud trabajo, en el análisis de las condiciones de salud y trabajo, inmersas un contexto epidemiológico, político, cultural y de la salud pública y laboral.

En el caso de Colombia se identifican estudios limitados que relacionan la tuberculosis como un problema de índole laboral en el sector salud. Si bien es reconocida la exposición a contaminantes biológicos en el sector salud (Decreto 2566 de 2009), se requiere la generación de investigaciones que involucren aspectos relacionados a la epidemiologia de casos a nivel laboral, estudios moleculares de infección latente, estudios de la transmisión de la infección en las instituciones de salud entre otros, que brinden relevancia a las acciones de prevención, identificación, vigilancia y control de la infección tuberculosa en especial, en hospitales donde se realiza el diagnóstico y atención a pacientes con la enfermedad.

Por su parte, la bibliometría como una herramienta que permite acercarse a los desarrollos investigativos en un determinado campo, cobra una inusitada importancia en los alcances de la investigación en la salud y seguridad en el trabajo en el sector salud y en particular en la problemática de exposición ocupacional al agente Mycobacterium Tuberculosis.

Finalmente, se verifica la necesidad de fomentar los estudios mediante el liderazgo de las instituciones académicas, hospitalarias, gremiales, que generen redes de investigación en tuberculosis, que permita orientar políticas que potencialicen el abordaje a la problemática relevante en la salud y seguridad en el trabajo, la construcción de entornos de trabajo saludables, camino que facilita la aplicación de la promoción de la salud en los lugares de trabajo y prevención de efectos adversos.

\section{AGRADECIMIENTOS}

Los autores agradecen a la Universidad Nacional de Colombia, a la Facultad de Enfermería y a la Maestría en Salud y Seguridad en el Trabajo, centros de excelencia académica e investigación. A nuestras familias por su invaluable apoyo.

\section{REFERENCIAS BIBLIOGRÁFICAS}

1. World Health Organization-WHO. Global Tuberculosis Control: Surveillance, planning, financing. WHO Report. 2011. ISBN 9789241564380 Disponible en http://www.who.int/tb/publications/global_ report/2011/gtbr11_full.pdf.

2. Fica C Alberto, Cifuentes D Marcela, Ajenjo H M. Cristina, Jemenao P M. Irene, Zambrano O Alejandra, Febré V Naldy et al . Tuberculosis en el personal de salud. Rev. chil. infectol. [serial on the Internet]. 2008 Aug [cited 2011 July 25] ; 25(4): 243-255. Available from: http://www.scielo.cl/scielo.php?script=sci_ arttext\&pid=S0716-10182008000400001\&lng=en. doi: 10.4067/S0716-10182008000400001. 
3. Shukla SJ, Warren DK, Woeltje KF, Gruber CA, Fraser VJ. Factors associated with the treatment of latent tuberculosis infection among health-care workers at a midwestern teaching hospital. Chest. 2002;122(5):1609-14. Available from: http://www.ncbi.nlm.nih.gov/pubmed/12426260.

4. Rumetshofer R. and Rowhani M. Preventing tuberculosis infection in the health-care setting - A review. Atemwegs- und Lungenkrankheiten 2008 34:10 (406-407) http://www.bases.unal.edu.co:5026/search/res ults?subaction $=$ viewrecord $\&$ rid $=18 \&$ page $=5 \&$ L 352718261 .

5. Organización Mundial de la Salud- OPS. Salud y Seguridad en el trabajo en el sector Salud. Manual para gerentes y administradores. Washington. 2005. ISBN 9275325820 Consultado en http://www.bvsde. ops-oms.org/ssmanual/Spanish/SaludYSeguridad1.pdf\%20.pdf.

6. Lien LT, Hang NTL, Kobayashi N, Yanai H, Toyota E, Sakurada S, et al. Prevalence and risk factors for tuberculosis infection among hospital workers in Hanoi, Viet Nam. Plos One. 2009;4(8):e6798-e. Disponible en http://www.plosone.org/article/info:doi\%2F10.1371\%2Fjournal.pone.0006798.

7. Ringshausen FC, Schlösser S, Nienhaus A, Schablon A, Schultze-Werninghaus G, Rohde G. In-hospital contact investigation among health care workers after exposure to smear-negative tuberculosis. Journal Of Occupational Medicine And Toxicology (London, England). 2009;4:11-. Disponible en http://www. sinab.unal.edu.co:2109/login.aspx?direct=true $\& \mathrm{db}=$ mnh\&AN=20653946\&lang=es $\&$ site=ehost-live.

8. Organización Panamericana de la Salud. Boletín Día Mundial de la Salud. Unidad de Recursos Humanos para la salud OPS, 2006.

9. Organización Internacional del Trabajo -OIT. Enciclopedia de Salud y Seguridad. Prevención Control y vigilancia de la Tuberculosis; 1998 Tercera edición.

10. O'Donnell MR, Jarand J, Loveday M, Padayatchi N, Zelnick J, Werner L, et al. High incidence of hospital admissions with multidrug-resistant and extensively drug-resistant tuberculosis among South African health care workers. Annals Of Internal Medicine. 2010;153(8):516-22 Disponible en http://www.sinab. unal.edu.co:2109/login.aspx?direct $=$ true $\& \mathrm{db}=$ mnh\&AN=20956708\&lang=es \&site $=$ ehost-live.

11. Betancourt, O. Texto para la enseñanza e investigación de la salud y seguridad en el trabajo Ed FUNSAD OPS/OMS; 1999, Quito. P.63.

12. Callon, Michel. Cienciometría: el estudio cuantitativo de la actividad científica de la bibliometría a la vigilancia tecnológica Gijón. 1995. 110 p.. 110 p http://www.scielo.org.ar/scieloOrg/php/reflinks. php?refpid=S1851-1740201000010000300005\&pid=S1851-17402010000100003\&lng=es.

13. Araujo Ruiz, Juan A Y Arencibia Jorge, Ricardo. Informetría, bibliometría y cienciometría: aspectos teórico-prácticos. ACIMED [online]. 2002, vol.10, n.4, pp. 5-6. ISSN 1024-9435. http://scielo.sld.cu/scielo. php?pid=S1024-94352002000400004\&script=sci_arttext\&tlng=pt.

14. Sterling TR, Haas DW. Transmission of Mycobacterium tuberculosis from health care workers. The New England Journal Of Medicine. 2006;355(2):118-21. http://www.sinab.unal.edu.co:2109/login.aspx?direct= true $\& \mathrm{db}=\mathrm{mnh} \& \mathrm{AN}=16837674 \&$ lang $=\mathrm{es} \&$ site $=$ ehost-live.

15. Özsoy S., Akar T., Gümüș S., Dinç A.H., Demirel B. and Safali M. The results of tuberculin skin test and the risk of tuberculosis in autopsyworkers. Turkiye Klinikleri Journal of Medical Sciences. 2010 30:6 (1876-1883) http://tipbilimleri.turkiyeklinikleri.com/abstract_59456.html.

16. Sawanyawisuth K, Chaiear N, Takahashi K, et al. Can workplaces be predictors for recent onset latent tuberculosis in health care workers?. Journal of Occupational Medicine And Toxicology (London, England) [serial online]. July 24, 2009;4:20. Available from: MEDLINE with Full Text, Ipswich, MA. Accessed June 9, 2012. http://www.biomedcentral.com/content/pdf/1745-6673-4-20.pdf.

17. García-García M.L., et al. Factors associated with tuberculin reactivity in two general hospitals in Mexico. Infection Control and Hospital Epidemiology. Mexico 2001: 22:2 (88-93) http://www.bases.unal.edu. co:5026/search/results?subaction=viewrecord\&rid=7\&page=1\&L32157158.

18. Roth Vr, Garrett Do, Laserson Kf, et at. A multicenter evaluation of tuberculin skin test positivity and conversion among health care workers in Brazilian hospitals. The Official Journal of The International Union Against Tuberculosis And Lung Disease. 2005. 9 (12), p 1335-42 ISSN: 1027-3719 http://www. curehunter.com/public/pubmed16466055.do.

19. López, Lillian K.O. Msc, RN a; Teles, Sheila A. PhD b; Souza, Adenicia C.S. Tuberculosis risk among nursing professionals from Central Brazil. AJIC: American Journal of Infection Control. 2008; 36(2):148151. http://www.ajicjournal.org/article/S0196-6553(07)00463-4/abstract.

20. Bonifacio N, Saito M, Gilman RH, Leung F, Cordova Chavez N, Chacaltana Huarcaya J, et al. High risk for tuberculosis in hospital physicians, Peru. Emerg Infect Dis 2002,8:747-748. http://www.scielo.cl/scieloOrg/ php/reflinks.php? refpid=S0716-1018200800040000100006\&pid=S0716-10182008000400001\&lng=es.

21. Louther J, Rivera P, Feldman J, Villa N, DeHovitz J, Sepkowitz KA. Risk of tuberculin conversion according to occupation among health care workers at a New York City hospital. Am J Respir Crit Care Med 1997,156:201-205. http://www.ncbi.nlm.nih.gov/pubmed/9230748. 
22. Ostrosky-Zeichner L, Rangel-Frausto S, García-Romero E et ál. Tuberculosis en trabajadores de la salud: importancia de los programas de vigilancia y control. Salud pública Méx. 2000; 42(1): 48-52 [ consultado el 11 de abril de 2010]. En: http://www.scielosp.org/pdf/spm/v42n1/2399.pdf.

23. Brock I, Weldingh K, Lillebaek T, Follmann F, Andersen P. Comparison of tuberculin skin test and new specific blood test in tuberculosis contacts. Am J Respir Crit Care Med. 2004 Jul 1;170(1):65-9. http:// ajrccm.atsjournals.org/content/170/1/65.short.

24. Soborg B, Andersen AB, Larsen HK, Weldingh K, Andersen P, Kofoed K, et al. Detecting a low prevalence of latent tuberculosis among health care workers in Denmark detected by $M$. tuberculosis specific IFNgamma whole-blood test. Scandinavian Journal Of Infectious Diseases. 2007;39(6-7):554-9. http://www. ncbi.nlm.nih.gov/pubmed/17577817.

25. Storla DG, Kristiansen I, Oftung F, Korsvold GE, Gaupset M, Gran G, et al. Use of interferon gammabased assay to diagnose tuberculosis infection in health care workers after short term exposure. BMC Infectious Diseases. 2009;9:60-. http://www.biomedcentral.com/1471-2334/9/60.

26. Arbeláez M, Ocampo MC, Montoyaj, Jaramillo LM, Giraldo, y otros. Evaluación de la respuesta a la tuberculina en estudiantes del área de la salud. Rev Panam Salud Publica vol.8 n.4 Washington Oct. 2000 http://www.scielo.org.co/scielo.php?script=sci_nlinks\&ref=000094\&pid=S0120$4157200400050001700017 \& \operatorname{lng}=\mathrm{en}$.

27. 27 Brudey K, Driscoll JR, Rigouts L, Prodinger WM, Gori A, Al-Hajoj SA, et al. Mycobacterium tuberculosis complex genetic diversity: mining the fourth international spoligotyping database (SpolDB4) for classification, population genetics and epidemiology. BMC Microbiol 2006,6:23. http://www.ncbi.nlm. nih.gov/pubmed/16519816.

28. Ashworth M, Horan K, Freeman R. Use of PCR-Based Mycobacterium tuberculosis Genotyping To Prioritize Tuberculosis Outbreak Control ActivitiesJ. Clin. Microbiol. 2008 46:856-862 http://www.ncbi. nlm.nih.gov/pmc/articles/PMC2268366/.

29. Bhanu N V, Banavalikar J N, Kapoor S K, Seth P. Suspected small-scale interpersonal transmission of Mycobacterium tuberculosis in wards of an urban hospital in Delhi, India. Am J Trop Med Hyg 2004; 70: 527-31. http://www.ncbi.nlm.nih.gov/pubmed/15155985.

30. Sanchéz Tarragó N. La comunicación de la ciencia en los países en vías de desarrollo y el Movimiento Open Access Biblios, 2007 - redalyc.uaemex.mx Lima, Peru. http://redalyc.uaemex.mx/src/inicio/ ArtPdfRed.jsp?iCve=16102703.

31. Franchi A, Richeldi L, Parrinello G, Franco G. Room size is the major determinant for tuberculin conversion in health care workers exposed to a multidrug-resistant tuberculosis patient. International Archives Of Occupational And Environmental Health. 2007;80(6):533-8. http://www.ingentaconnect. com/content/klu/420/2007/00000080/00000006/00000160.

32. Velayati, P. Farnia, M.R. Masjedi, T.A. Ibrahim, P. Tabarsi, R.Z. Haroun, H.O. Kuan, J. Ghanavi, P. Farnia and M. Varahram, "Totally drug-resistant tuberculosis strain: evidence adaptation at the cellular level", European Respiratory Journal, 34,2009, 1202-1203. http://e-kube.com/blog/tdr-tb-totally-drugresistant-tuberculosistuberculosis-resistente-a-todos-los-medicamentos/.

33. Rafiza S; Rampal KG; Tahir A. Prevalence and risk factors of latent tuberculosis infection among health care workers in Malaysia. England; 2011. BMC Infectious Diseases http://ukpmc.ac.uk/articles/ PMC3033828/?report=abstract.

34. Sepkowitz K A Eisenberg L. Occupational deaths among healthcare workers. Emerg Infect Dis 2005; 11: 1003-8. http://www.ncbi.nlm.nih.gov/pubmed/16022771.

35. Murray M, Oxlade O, Lin HH. Modeling social, environmental and biological determinants of tuberculosis. Int J Tuberc Lung Dis. Massachusetts. 2011 Jun;15 Suppl 2:64-70. Disponible en http://www.ncbi.nlm.nih. gov/pubmed/21740661.

36. Muñoz AI, Lozada MA. Experiencias de Investigación en Salud y Seguridad en el Trabajo. Universidad Nacional de Colombia, Vicerrectoría de Investigación Sede Bogotá. Primera Edición 148p. ISBN 978-958761-172-4.

37. Nery de, Bertolozzi Maria Rita. La vulnerabilidad a la tuberculosis en trabajadores de enfermería dentro de un hospital universitario. Rev. Latino-Am. Enfermagem [serial on the Internet]. 2007 Apr [cited 2011 July 28]; 15(2): 259-266. Available from: http://www.scielo.br/scielo.php?script=sci_arttext\&pid=S0104.

38. Riley R L, Nardell E A. Clearing the air. The theory and application of ultraviolet air disinfection. Am Rev Respir Dis 1989; 139: 1286-94. http://www.ncbi.nlm.nih.gov/pubmed/2653151.

39. Menzies D, Fanning A, Yuan L, FitzGerald JM. Hospital ventilation and risk for tuberculous infection in canadian health care workers. Canadian Collaborative Group in Nosocomial Transmission of TB. Ann Intern Med 2000,133:779-789. http://www.annals.org/content/133/10/779.full.pdf.

40. Menzies D, Joshi R, Pai M. Risk of tuberculosis infection and disease associated with work in health care settings. Int J Tuberc Lung Dis 2007,11:593-605. http://www.ncbi.nlm.nih.gov/pubmed/17519089. 
41. Escombe AR, Moore DAJ, Gilman RH, Navincopa M, Ticona E, et al. Upper-Room Ultraviolet Light and Negative Air Ionization to Prevent Tuberculosis Transmission. PLoS Med 6(3):2009 e1000043. doi:10.1371/ journal.pmed.1000043.

42. Cruz OA, Flórez ÉL, Muñoz AI. Conocimientos sobre tuberculosis en trabajadores de la salud en una localidad de Bogotá D. C. av.enferm. [serial on the Internet]. 2011 July [cited 2012 May 28] ; 29(1): 143-151. Available from: http://www.scielo.org.co/scielo.php?script=sci_arttext\&pid=S0121-45002011000100014\&lng=en.

43. Laraqui CH, Laraqui O, Douma A, Mounassif M. and Rahhali A. Perception of occupational hazards among health care workers in prison in Morocco. Archives des Maladies Professionnelles et de Medecine du Travail 2002 63:5 (374-381).

44. World Health Organization, Center Diseases Control. Implementing the Policy on TB Infection. Control in Health-Care Facilities, Congregate Settings and Households A framework to plan, implement and scale-up TB infection control activities at country, facility and community level. 2009. Disponible en http://www.tbcta.org/Uploaded_files/Zelf/TBICImpFramework1305387835.pdf.

45. Muñoz AI, Lozada MA, Molano JH, Riaño MI, Castro E, Benavides JA. Experiencias de investigación en Salud y Seguridad en el Trabajo. Maestría en Salud y Seguridad en el trabajo-Universidad Nacional de Colombia Facultad de Enfermería. Colombia 2012. ed:Universidad Nacional de Colombia ISBN: 978-958761-170-0.

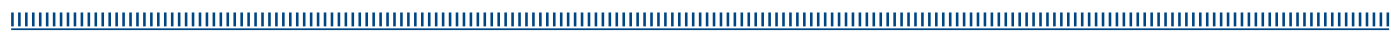

